\title{
Three cases of community-associated meticillin-resistant Staphylococcus aureus infection in China
}

The spread of meticillin-resistant

Staphylococcus aureus (MRSA) in the community has challenged infection control recently since it can be acquired by patients without established risk factors for MRSA infection and can lead to a fatal outcome. Such community-associated MRSA (CAMRSA) has been observed in many parts of the world (Hsu et al., 2005; Aires de Sousa et al., 2003; Ho et al., 2004). Three cases of CA-MRSA in mainland China and the genetic characteristics of the strains are described here in detail.

Case 1: In September 2006, a 36-year-old woman was admitted to the West China Hospital with a 4-day history of a carbuncle on the back of her foot. Pus from the lesion site was sent for culture immediately. MRSA was isolated; it was susceptible to ciprofloxacin, gentamicin, rifampicin, co-trimoxazole and vancomycin. The patient was treated with levofloxacin and metronidazole intravenously. Gentamicin was also used on the lesion area. The patient was previously healthy without any fundamental diseases or recent hospitalization.

Case 2: In February 2007, a 39-year-old Japanese man was brought to our hospital because of an abscess in the centre of his left armpit. About 1 week before admission, he got the infection in his armpit after swimming in Thailand but was not treated there. The bacterial culture of pus was performed in West China Hospital. Results showed a pure growth of MRSA, which was susceptible to gentamicin, rifampicin, clindamycin, co-trimoxazole, tetracycline and vancomycin. He was treated with vancomycin. This patient was strong and healthy without any history of hospitalization, surgery or residence in medical settings for the previous year.

Case 3: In April 2007, a 43-year-old man was admitted to our hospital because of a progressive pain and abscess in his finger.
The tip of the finger was necrotic. Secretion from the lesion site was cultured and amputation was performed. MRSA was isolated and found to be susceptible to ciprofloxacin, gentamicin, rifampicin, co-trimoxazole and vancomycin. The patient was treated with ciprofloxacin intravenously. He was previously healthy without any established risk factors for health-care-associated MRSA infection.

The MRSA strains from these three cases were named s35301, s19165 and s29635 sequentially. They were confirmed to be S. aureus by colony morphology, production of coagulase, as well as catalase tests. Resistance to meticillin was verified by both the cefoxitin disc diffusion test recommended by CLSI and multi-PCR of $m e c A / f e m B$ genes as described previously (CLSI, 2005; Jonas et al., 1999). Genes encoding Panton-Valentine leukocidin (PVL) were co-amplified with primers described elsewhere (Lina et al., 1999). Staphylococcus chromosome cassette mec (SCCmec) types were investigated by the novel multiplex PCR strategy described previously (Zhang et al., 2005). Genetic backgrounds of the three organisms were characterized further by PFGE, multilocus sequence typing (MLST) and staphylococcus protein A (spa) typing as described previously (Murchan et al., 2003; Enright et al., 2000; Aires de Sousa \& de Lencastre, 2003).

Two strains (s35301 and s19165) out of the three were positive for PVL. All three strains carried the type IVa SCCmec. Patterns with four or more different bands were designated separate PFGE types. They showed three different PFGE patterns, and clustered in two clones by MLST and spa typing (Fig. 1). Strains from case 1 and case 3 (s35301 and s29635) were ST59-t437. Both were isolated from the local Chinese people in Chengdu, a city in west China. The MRSA strain of case 2 was typed as ST8-t008. This strain had a unique PFGE pattern which was similar to that of the USA300 clone.
ST59 was considered to be a community clone in Taiwan and has spread to San Francisco, USA (Aires de Sousa et al., 2003; Huang et al., 2006). It is very different in genotype from the present five MRSA clone complex (CC5, CC8, CC22, CC30 and CC45) (Enright et al., 2002). Five meticillinsusceptible $S$. aureus (MSSA) strains from the UK and the USA and six MRSA strains from the USA, Taiwan, Sweden and Singapore also belong to ST59. Two ST59 MRSA strains from Singapore have been specified as community-acquired possessing the type V SCCmec (from http:// www.mlst.net). S. aureus of $\mathrm{t} 437$ has spread in some European countries such as Sweden, Norway, Germany, Denmark and others, and is also common in Taiwan. So far, 11 strains of $t 437$ from Norway have been typed as ST59 (from http:// www.ridom.de/spaserver). The two ST59 MRSA strains in our study may be from the local ST59 MSSA which acquired the type IVa SCCmec horizontally. However, the genetic background of MSSA in our region has not been surveyed. The possibility of ST59 MRSA input by foreign carriers can not be excluded.

Strain s19165 in our study was ST8-t008, possessing the type IVa SCCmec, with positive detection of the PVL gene, which showed the same genetic characteristics as those of the USA300 clone (King et al., 2006; Moran et al., 2006). This clone is mainly prevalent in the USA and some European countries, such as the UK, Netherlands, Germany, Austria and others, and it has also been found in Japan (from http://spa.ridom.de/spaserver), but it has not been found in mainland China before. This strain was isolated from a Japanese tourist who got his armpit infection when travelling in Thailand, which suggests that USA300 may have been prevalent there and was carried to west China.

All three strains can be considered community-associated by referring to the clinical information. Laboratory results 


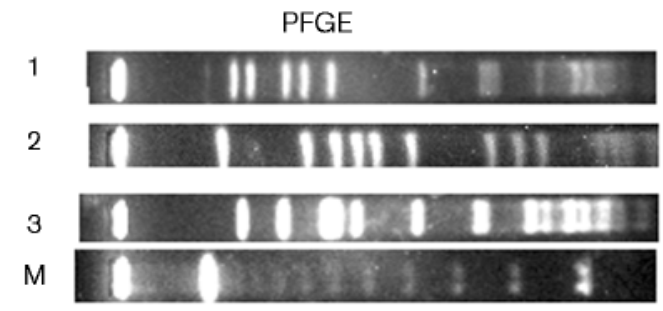

MLST type (allelic profile)

ST59 (19-23-15-2-19-20-15)

ST8 (3-3-1-1-4-4-3)

ST59 (19-23-15-2-19-20-15) spa type (repeat sequence)

t437 (04-20-17-20-17-25-34)

t008 (11-19-12-21-17-34-24-34-22-25)

t437 (04-20-17-20-17-25-34)

Fig. 1. Genetic types of the three CA-MRSA isolates. 1, s35301 (case 1); 2, s19165 (case 2); 3, s29635 (case 3); M, marker.

also characterized them as CA-MRSA: susceptible to several non- $\beta$-lactams, carriage of SCCmec type IVa, positive for PVL genes (two of them), and shown to be the same as CA-MRSA clones prevalent in other parts of the world by MLST and spa typing (Davis et al., 2007). Conclusively, CA-MRSA (SCCmecIVa-ST59-t437 and SCCmec IVa-ST8-t008) has been present in mainland China. It should be paid much attention because of the probable fatal results and potential for outbreak.

Screening patients with risk factors for CAMRSA might be useful for controlling its domestic spread in communities. Patients who have a history of travelling abroad should be screened for MRSA carriage before admittance to hospitals.

\section{Acknowledgements}

This work was supported by the InfectionManagement Department of West China Hospital. National Natural Science Foundation of China (NSFC: 30470761).

\section{Hong Fan, ${ }^{1,2} †$ Nan Li† and Lanlan Wang ${ }^{1}$ \\ ${ }^{1}$ Department of Laboratory Medicine, State Key Laboratory of Biotherapy, West China Hospital, Si Chuan University, PR China \\ ${ }^{2}$ Laboratory of Respiration Medicine, State Key Laboratory of Biotherapy, West China Hospital, Si Chuan University, PR China \\ tThese authors contributed equally to this paper.}

Correspondence: Hong Fan

(fanhongprof@gmail.com)
Aires de Sousa, M. \& de Lencastre, H. (2003). Evolution of sporadic isolates of methicillinresistant Staphylococcus aureus (MRSA) in hospitals and their similarities to isolates of community-acquired MRSA. J Clin Microbiol 41, 3806-3815.

Aires de Sousa, M., Crisóstomo, M. I., Sanches, I. S., Wu, J. S., Fuzhong, J., Tomasz, A. \& de Lencastre, H. (2003). Frequent recovery of a single clonal type of multidrug-resistant Staphylococcus aureus from patients in two hospitals in Taiwan and China. J Clin Microbiol 41, 159-163.

CLSI (2005). Performance Standards for Antimicrobial Susceptibility Testing; 15th Information Supplement, M100-S15. Wayne, PA: Clinical and Laboratory Standards Institute.

Davis, S. L., Perri, M. B., Donabedian, S. M., Manierski, C., Singh, A., Vager, D., Haque, N. Z., Speirs, K., Muder, R. R. \& other authors (2007). Epidemiology and outcomes of communityassociated methicillin-resistant Staphylococcus aureus infection. J Clin Microbiol 45, 1705-1711.

Enright, M. C., Day, N. P. J. \& Davies, C. E. (2000). Multilocus sequence typing for characterization of methicillin-resistant and methicillin-susceptible clones of Staphylococcus aureus. J Clin Microbiol 38, 1008-1015.

Enright, M. C., Robinson, D. A., Randle, G., Feil, E. J., Grundmann, H. \& Spratt, B. G. (2002). The evolutionary history of methicillin-resistant Staphylococcus aureus (MRSA). Proc Natl Acad Sci U S A 99, 7687-7692.

Ho, P. L., Tse, C. W. S., Mak, G. C., Chow, K. H. \& $\mathrm{Ng}, \mathrm{T}$. K. (2004). Community-acquired methicillin-resistant Staphylococcus aureus arrives in Hong Kong. J Antimicrob Chemother 54, 845-846.

Hsu, L. Y., Tristan, A., Koh, T. H., Bes, M., Etienne, J., Kurup, A., Tan, T. T. \& Tan, B. H. (2005). Community-associated methicillinresistant Staphylococcus aureus, Singapore. Emerg Infect Dis 11, 341-342.

Huang, Y. C., Su, L. H., Wu, T. L. \& Lin, T. Y. (2006). Changing molecular epidemiology of methicillin-resistant Staphylococcus aureus bloodstream isolates from a teaching hospital in Northern Taiwan. J Clin Microbiol 44, 2268-2270.

Jonas, D., Grundmann, H., Hartung, D., Daschner, F. D. \& Towner, K. J. (1999). Evaluation of the mecA femB duplex polymerase chain reaction for detection of methicillinresistant Staphylococcus aureus. Eur J Clin Microbiol Infect Dis 18, 643-647.

King, M. D., Humphrey, B. J., Wang, Y. F., Kourbatova, E. V., Ray, S. M. \& Blumberg, H. M. (2006). Emergence of community-acquired methicillin-resistant Staphylococcus aureus USA 300 clone as the predominant cause of skin and soft-tissue infections. Ann Intern Med 144, 309-317.

Lina, G., Piémont, Y., Godail-Gamot, F., Bes, M., Marie-Odile, P., Gauduchon, V., Vandenesch, F. \& Etienne, J. (1999). Involvement of PantonValentine leukocidin producing Staphylococcus aureus in primary skin infections and pneumonia. Clin Infect Dis 29, 1128-1132.

Moran, G. J., Krishnadasan, A., Gorwitz, R. J., Fosheim, G. E., McDougal, L. K., Carey, R. B. \& Talan, D. A. (2006). Methicillin-resistant Staphylococcus aureus infections among patients in the emergency department. N Engl J Med 355, 666-674.

Murchan, S., Kaufmann, M. E., Deplano, A., de Ryck, R., Struelens, M., Zinn, C. E., Fussing, V., Salmenlinna, S., Vuopio-Varkila, J. \& other authors (2003). Harmonization of pulsed-field gel electrophoresis protocols for epidemiological typing of strains of methicillin-resistant Staphylococcus aureus: a single approach developed by consensus in 10 European laboratories and its application for tracing the spread of related strains. J Clin Microbiol 41, 1574-1585.

Zhang, K., McClure, J. A. \& Elsayed, S. (2005). Novel multiplex PCR assay for characterization and concomitant subtyping of staphylococcal cassette chromosome mec types I to $\mathrm{V}$ in methicillin-resistant Staphylococcus aureus. J Clin Microbiol 43, 5026-5033. 